\title{
Magnetic Fields in Filaments
}

\section{P. Démoulin}

Observatoire de Paris, Section de Meudon, DASOP, URA 2080 (CNRS) F-92195 Meudon Cedex, France

\begin{abstract}
Filaments are present in highly non-potential magnetic configurations. On one hand, the complexity of modeling such $3-\mathrm{D}$ configurations makes a useful comparison between observations and models difficult. On the other hand such highly sheared regions are more interesting and challenging for understanding eruptive phenomena like flares and coronal mass ejections. Fortunately, the presence of cold plasma allows us to measure the magnetic field inside prominences. Together with photospheric field measurements and other morphological observations, these provide a large set of puzzling constraints for plausible models of the magnetic configurations. Models are reviewed in the framework of present observational constraints with the aim to clarify a piece of the mystery which surrounds the magnetic configuration of filaments.
\end{abstract}

\section{Observational and Theoretical Constraints}

The magnetic field plays a key role in all the processes involved in the corona as in prominences because the plasma beta is low (in the range $10^{-3}-10^{-1}$ ). It channels both the plasma motions and the thermal conduction. It provides support against gravity of the prominence plasma one hundred times denser than the coronal plasma. This primary role is not always recognized at its right level because the majority of observations are focused on the plasma. Furthermore, evidences for importance of the magnetic-field are even less compelling in prominences than in other phenomena since the prominence plasma do not usually visualize field lines as it does in arch-filament systems, surges and coronal loops. Models for the magnetic configurations are also difficult to build, both because the configurations are strongly non-potential and because even the global shape (topology) of the configurations is unknown.

Under these circumstances the detective story, started few decade ago, is still alive. We have now many indices: photospheric magnetograms, magnetic measurements inside prominences as well as the pattern of chromospheric fibrils and coronal loops. Still there are several theoretical scenarios and some observations provide alibi for some accused magnetic configurations. As in every good detective story, some of the alibis should be false; but which ones? Below I present my personal and actual (!) view on this interesting story and try to glue together as many pieces of the puzzle as possible. 


\subsection{Results from Magnetic-Field Measurements}

Prominences are thin structures consisting of cold plasma embedded in the hot corona. Their global shape has been known for a long time (e.g., d'Azambuja and d'Azambuja 1948). They are always found above inversion lines of the photospheric magnetic field in regions, called corridors or filament channels, which are nearly free of chromospheric fibrils (Martres et al. 1966). They are also characterized on either side by the presence of $\mathrm{H} \alpha$ fibrils nearly aligned with the inversion line, indicating a high magnetic shear (Foukal 1971, Rompolt 1990). The corridor is nearly free of vertical magnetic field flux except small parasitic polarities (Martin 1990). A necessary condition for prominence formation is a low gradient, transverse to the photospheric inversion line, of the vertical field component (Shelke and Pande 1983, Maksimov and Ermakova 1985, Maksimov and Prokopiev 1995).

In prominences the Zeeman effect only allows the measurement of the longitudinal component of the magnetic field (see Kim 1990, and references therein). Radio wavelengths provide information on the field strength (e.g., Apushkinskii et al. 1990). The Hanle effect gives the three components of the field (and the electron density) from the polarization measurements in two spectral lines (e.g., Bommier et al. 1994). The compatibility of the results obtained by these three independent methods and by different groups of observers have strongly validated the results (see Leroy 1988, 1989, and Kim 1990).

One of the main results of Hanle measurements is that the prominence field has the opposite direction to that expected from extrapolation of photospheric measurements (e.g., Leroy et al. 1983). Not only the field component orthogonal to the prominence is opposite to the field of a simple arcade (referred to inverse configuration), but also the field component parallel to the prominence is opposite to those of an arcade that would have been sheared by differential rotation! This has been shown after a detailed analysis because twin solutions, symmetrical with regard to the line of sight, exist with optically thin lines and right angle scattering (known as the $180^{\circ}$ ambiguity). In the majority of cases, one solution is normal and the other inverse. At the beginning of the studies only the normal solution was retained (before 1983). But it became increasingly clear that this was in fact the wrong solution. This ambiguity has been resolved in four different ways: by statistical studies, by selecting unambiguous cases, by following the perspective evolution during a few days and, recently, by using a marginally thick line. A large majority of prominences belong to the inverse type $(75 \%$ in Leroy et al. $1984,85 \%$ in Bommier et al. 1994 and greater than $90 \%$ in Bommier and Leroy 1998, these proceedings).

It is now well accepted that the magnetic field in prominences is nearly horizontal (since Athay et al. 1983), while compatible with a slight magnetic dip (Bommier et al. 1986, 1994). The magnetic field strength is nearly homogeneous (Leroy 1989) on the scale of a few arc seconds, but shows a statistical increase of strength with height which is compatible with a dip configuration (e.g., Leroy et al. 1983).

\subsection{Why Is a Magnetic Dip Required?}

The gravitational scale height of prominence material $(\approx 200-500 \mathrm{~km})$ is much lower than the typical height of prominences $\left(\approx 10^{4}-10^{5} \mathrm{~km}\right)$. This implies 
that plasma pressure cannot provide the mechanism of support. The observed velocities (few $\mathrm{km} \mathrm{s}^{-1}$ ) are much less important than the free-fall velocities (several $10 \mathrm{~km} \mathrm{~s}^{-1}$ ) and, usually, the velocity pattern does not show a pattern similar to the one seen in arch filament systems where plasma is clearly seen flowing down (Schmieder 1990). Thus the cold prominence plasma should be supported by the magnetic field. If enough mass (i.e., a plasma beta greater than 0.05 ) can be brought exactly to the top of sheared magnetic arcade in less than one hour, the gravity force can bend down the top of the field lines, providing a stable support for the prominence plasma (Schmieder et al. 1991, Fiedler and Hood 1993). However prominence formation takes usually a few days (resp. few weeks) in (resp. outside) active regions (e.g., Malherbe 1989). This implies that the magnetic dip should be intrinsic to the magnetic configuration (rather than being formed by plasma forces).

\subsection{Observed Evidence for Magnetic Dips}

Finding observational support for the existence of magnetic dips in prominences is not trivial. But the dip is not incompatible with the observed vertical fine structures (see, for example, Poland 1990, Steele and Priest 1992). The cold plasma fills only the extreme lower part of the field lines (over a height typically one hundred times lower than the field line radius of curvature). Therefore, the effect is too shallow to be observed in the quiescent phase. Higher spatial resolution does not really help because one can only observe the mixing of several fine structures along the line of sight. In the quiescent phase, the presence of magnetic dips is only shown indirectly by the Hanle measurements (e.g., Bommier et al. 1994).

Observational evidence for dips is present directly only in some eruptions (e.g., Rompolt 1990). Several favorable conditions should be present together for direct observation of dips: First, a spatial resolution good enough to see individual filled-flux tubes; second, a quantity of cold plasma sufficient to observe it, but not too great so that there are not too many overlapping structures! Finally, the magnetic dip should change location, (which implies a plasma flow from the old to the new dip location), otherwise the prominence plasma should be accelerated along field lines (e.g., like in surges).

\section{Magnetic Configurations Supporting Dense Plasma}

The need of a magnetic dip for stable support was first pointed out by Kippenhahn and Schlüter (1957), however, the implications of such a condition were used only recently to select magnetic configurations suitable for prominence formation (see Priest et al. 1989).

\subsection{Prominences Supported in Arcade-Like Configurations}

A dip cannot be present in a force-free $2 \frac{1}{2} \mathrm{D}$ arcade, since the field lines become flatter as the magnetic shear increases (Amari et al. 1991). This is however possible in 3-D with an overlaying arcade compressing locally the central-part of an underlying sheared arcade (Antiochos et al. 1994). The latter gives mostly an inverse-polarity prominence with a magnetic field nearly aligned with the photospheric inversion line. 


\subsection{Prominence Supported in Twisted-Flux Tubes}

Another way to create a dip is to form a twisted magnetic configuration. It can be formed in several ways: by photospheric twisting motions (Priest et al. 1989), by converging motions in a sheared arcade with magnetic reconnection at the inversion line (van Ballegooijen and Martens 1989), by resistive instability in a sheared arcade (e.g., Inhester et al. 1992), by relaxation and accumulation of helicity (e.g., Démoulin and Priest 1989, Amari and Aly 1992, Rust and Kumar 1994), or by emergence from the convective zone (e.g., Low 1994 1996).

Many observations suggest a helical-like pattern during eruption of prominences: on the disk (e.g., Raadu et al. 1988) and more often at the limb (e.g., House and Berger 1987, Rompolt 1990, Srivastava et al. 1991, Vršnak et al. 1991). Twisted configurations are also identified in CMEs with in situ measurements from Ulysses (e.g., Bothmer et al. 1996, Weiss et al. 1996). Even in the quiescent phase, Filippov (1994) discovered a pattern typical of twisted flux tubes: a "fishbone" organization in $\mathrm{H} \alpha$ fibrils on both sides of the inversion line. Low and Hundhausen (1995) show how a twisted-flux tube topology can bring together many of the $\mathrm{H} \alpha$ and magnetic observations. Finally, the emergence of a twisted configuration is supported by recent vector field measurements (Leka et al. 1996, Lites et al. 1995).

\subsection{Prominences Supported in Quadrupolar Configurations}

Quadrupolar configurations naturally have a dip. Kippenhahn and Schlüter (1957, their section 4) first proposed these inverse configurations for stable support of dense plasma; curiously very few authors have referred to this part of their work. Then Uchida (1981) and Malherbe and Priest (1983) independently proposed this configuration; the community remained mostly skeptical. The model was further developed by Démoulin and Priest (1993), Drake et al. (1993), and Uchida (1998, these proceedings). The presence of a corridor free of significant field is needed to have a prominence extension reaching the chromosphere and converging motions to provide mass supply. The quadrupolar model has been extended to magnetic configurations typically found in active regions (Titov et al. 1993, Bungey et al. 1996) and in polar crown regions (Cartledge et al. 1996). Finally, the equilibrium of a twisted flux tube in a quadrupolar region gives a normal polarity prominence (Amari and Aly 1992, Démoulin and Raadu 1992, Lepeltier and Aly 1994), while all other models above have an inverse polarity.

The most important observational supports are the following. First, $\approx 65 \%$ of prominences lie in between bipolar regions (Tang 1987). Second, the long-term effect of differential rotation on them leads to the observed magnetic direction in prominences (see Bommier and Leroy 1998, these proceedings). Finally the presence of "dual arcades" in Yohkoh observations is a signature of magnetic reconnection in quadrupolar configurations (Uchida 1998, these proceedings).

\subsection{Common Features of Models with Dips}

In the vicinity of prominences, models with magnetic dips have the following common and observed properties (see section 1.1):

- the field strength increases with height;

- a low vertical magnetic flux is present in the corridor;

- the field component crossing the prominence is inverse; 
- the emergence of flux implies converging, canceling fluxes, and lift-up of dense plasma.

I then deduce that the latter should be realized in prominence feet; it is then natural that the feet are observed to form first (Martres et al. 1966). Moreover, magnetic reconnection is required to progressively liberate the magnetic configuration from its deep anchorage (Low and Hundhausen 1995).

\section{Large-Scale Magnetic Field Organization}

\subsection{Magnetic-Field Observations}

There is a global spatial organization and a temporal evolution in phase with the solar cycle of the field measured in prominences (Leroy 1989). In particular, the field orientation is generally continuous along inversion lines. The direction of the field reverses only if one goes to the next inversion line or if one changes hemisphere. Moreover, the tilt of field direction along the equator is nicely correlated with the differential rotation but with a sign opposite to a sheared arcade (see Bommier and Leroy 1998, these proceedings).

\subsection{Chirality Patterns}

The magnetic observations are coherent with a segregation of chirality by hemisphere. For the northern hemisphere:

- The magnetic helicity is negative (Seehafer 1990, Pevtsov et al. 1995);

- The filaments are dextral and right bearing (Martin et al. 1994);

- The overlaying coronal arcade is left-bearing (Martin and Mc Allister 1996); and the opposite for the southern hemisphere.

\subsection{Proposed Scenarios}

A natural way to explain that both magnetic field components (parallel and normal to the prominence) are inverse is to use a quadrupolar field sheared by the differential rotation. However, this gives a magnetic helicity dominance in each hemisphere opposite to observations. The right helicity sign is obtained with Coriolis forces acting on diverging supergranular flows. Since it is typically five times more efficient than differential rotation (Priest et al. 1989), it can lead to accumulation of helicity in twisted flux tubes. However, localized twisting motions give neutralized currents while a dominance of one polarity is observed in each hemisphere (e.g., Pevtsov et al. 1995). Van Ballegooijen and Martens (1990) instead proposed the effect of differential rotation in sub-photospheric layers. Then the sheared field becomes buoyant and emerges forming a twisted flux tube with both field components inverse at the dip locations. Recently, Kuperus (1996) proposed a coronal process in which the presence of discrete flux tubes is fundamental (together with converging motions and magnetic reconnection). In conclusion, while the field component orthogonal to the prominence is almost always inverse in models in which one imposes a dip (section 2.4), the fact that the field component parallel to the prominence is also inverse is not yet understood. It is likely that this is caused by the global dynamo (see Berger 1998, Seehafer 1998, both these proceedings). 


\section{What Is Next?}

\subsection{Some Suggestions for Future Observations}

Looking at the observed fine structure in $\mathrm{H} \alpha$, one would like to see fine spatial details in the magnetic field, thus, an obvious improvement in magnetic measurements would be an increase of spatial resolution. However, in my view, this is not a major one because it will only reveal a small modulation associated with fine structures. Of much greater interest is to improve the polarimetry to deduce not only the vector magnetic field but its matrix gradient. Only in this way can observations put further constraints on models, in particular by selecting from different models. For example, the vertical variation of the angle between the prominence axis and the magnetic field is different in quadrupolar and in twisted models (in the first case the absolute value of this angle increases monotonically with height, while in the second it has a maximum in the middle of the dip region).

\subsection{Combination of Photospheric and Prominence Measurements}

The main way to deduce coronal magnetic fields is via extrapolation from photospheric magnetograms. Measurements in prominences give more direct clues on coronal fields, but they are localized ones and are not sufficient for computing the whole coronal configuration. While these data are not co-temporal (disk and limb observations) one can still try to combine them because of the slow time evolution of the field supporting quiescent prominences ( $\geq$ weeks, Leroy 1989). In this way one can expect to create a better model for the coronal field.

The above mixed-boundary problem was first introduced by Anzer (1972) for a massive and vertical current sheet embedded in the 2-D potential case. It models a prominence attached to the chromosphere. The generalization to a detached prominence was made by Démoulin et al. (1989). The presence of mixed-boundary conditions brings several problems. Aly et al. (1989) and Démoulin and Forbes (1992) removed both unphysical line current singularities and lack of support at the prominence bottom and top. Then two regularization procedures were proposed by Démoulin and Priest (1993) and Lepeltier and Aly (1995) to apply the method to real data. One extension is to have a finite prominence width by matching an internal solution to the global one (Cartledge and Titov 1996). Another extension to a constant-current (linear) force-free field was done by Ridgway et al. 1992 (Démoulin and Raadu 1992).

To extend the aforementioned work to more general fields looks realisable in $2 \frac{1}{2}$-D (one step in this direction is the work of Low and Hundhausen 1995), but there is presently no known way to generalize this to 3-D configurations. The only promising way is the extrapolation of photospheric data (without taking into account the presence of prominence material). This is justified to a first approximation by the low plasma $\beta$. There are recent advances in the extrapolation methods: see Amari et al. (1997) and Jiao et al. (1997). One can then compare the extrapolated magnetic field to the one measured in the prominences present in the region. If there is a rough agreement, one then can add cold material, with the observed density, and see if it can improve the correspondence. This is obviously not an easy task, because of the limitations of the extrapolation methods but also because of noise present in the transverse field measurements. 
We are hoping that new vector magnetographs, in particular the Franco-Italian THEMIS telescope (e.g., Molodij et al. 1996), will improve the measurements. Finally, the prominence field measurements will put important constraints on magnetic extrapolation and help to construct realistic coronal fields. This is important to understanding prominence physics but it also has an impact on the determination of the global solar magnetic field with direct implications on dynamo theories and solar activity.

Acknowledgments. I thank V. Bommier and L. van Driel Gesztelyi for their help to improve the manuscript.

\section{References}

Aly J.J., Amari T., Colombi S. 1989, in M.A. Dubois, F.Bely-Dubau, D.Gresillon (eds.), Les Editions de la Physique, p. 181

Amari T., Aly J.J. 1992, A\&A, 265, 791

Amari T. et al. 1997, Solar Phys., 174, 129

Amari T., Démoulin P., Browning P., Hood A.W., Priest E.R. 1991, A\&A, 241, 604

Antiochos S.K., Dahlburg R.B., Klimchuck J.A. 1994, ApJ, 420, L41

Anzer U. 1972, Solar Phys., 24, 324

Apushkinskii G.P., Nesterov N.S., Topchilo N.A., Tsyganov A.N. 1990, SvA, 34(5), 530

Athay R.G. et al. 1983, Solar Phys., 89, 3

Bommier V., Leroy J.L., Sahal-Bréchot S. 1986, A\&A, 156, 79

Bommier V. et al. 1994, Solar Phys., 154, 231

Bothmer V. et al. 1996, A\&A, 316, 493

Bungey T.N., Titov V.S., Priest E.R. 1996, A\&A, 308, 233

Cartledge N.P., Titov V.S. 1996, Solar Phys., 169, 55

Cartledge N.P., Titov V.S., Priest E.R. 1996, Solar Phys., 166, 287

d'Azambuja L., d'Azambuja M. 1948, Annales de l'Observatoire de Paris, 6, 7

Démoulin P., Forbes T.G. 1992, ApJ, 387, 394

Démoulin P., Priest E.R. 1989, A\&A, 214, 360

Démoulin P., Priest E.R. 1993, Solar Phys., 144, 283

Démoulin P., Raadu M.A. 1992, Solar Phys., 142, 291

Démoulin P., Malherbe J.M., Priest E.R. 1989, A\&A, 211, 428

Drake J.F., Mok Y., van Hoven G. 1993, ApJ, 413, 416

Fiedler R.A.S., Hood A.W. 1993, Solar Phys., 146, 297

Filippov B.P. 1994, Astron. Letters, 20, 665

Foukal P. 1971, Solar Phys., 19, 59

House L.L., Berger M.A. 1987, ApJ, 323, 406

Inhester B., Birn J., Hesse M. 1992, Solar Phys., 138, 257

Jiao L., McClymont, A.N. and Mikić, Z. 1997, Solar Phys., 174, 311

Kim I.S. 1990, Dynamics of Quiescent Prominences, Springer-Verlag, (eds.) V. Ruždjak, E. Tandberg-Hanssen, p. 49

Kippenhahn R., Schlüter A. 1957, Zs. Ap. 43, 36

Kuperus M. 1996, Solar Phys., 169, 349

Leka K.D., Canfield R.C., McClymont A.N., van Driel-Gesztelyi L. 1996, ApJ, 462, 547

Lepeltier T., Aly J.J. 1994, Solar Phys., 154, 393

Lepeltier T., Aly J.J. 1995, A\&A, 293, 906

Leroy J.L. 1988, in Solar and Stellar Coronal Structures and Dynamics, R.C. Altrock 
(ed.), National Solar Observatory, Sunspot, NM, p. 422

Leroy J.L. 1989, in Dynamics and Structure of Quiescent Solar Prominences, E.R. Priest

(ed.), Kluwer Acad. Publ., Dordrecht, Holland, p. 77

Leroy J.L., Bommier V., Sahal-Bréchot S. 1983, Solar Phys., 83, 135

Leroy J.L., Bommier V., Sahal-Bréchot S. 1984, A\&A, 131, 33

Lites B.W. et al. 1995, ApJ, 446, 877

Low B.C. 1994, Plasma Phys., 1, 1684

Low B.C. 1996, Solar Phys., 167, 217

Low B.C., Hundhausen J.R. 1995, ApJ, 443, 818

Maksimov V.P., Ermakova L.V. 1985, SvA, 29(3), 323

Maksimov V.P., Prokopiev A.A. 1995, Astron. Nachr., 4, 249

Malherbe J.M. 1989, in Dynamics and Structure of Quiescent Solar Prominences, E.R. Priest (ed.), Kluwer Acad. Publ., Dordrecht, Holland, p. 115

Malherbe J.M., Priest E.R. 1983, A\&A, 123, 80

Martin S.F. 1990, in Dynamics of Quiescent Prominences, V. Ruždjak, E. TandbergHanssen (eds.), Springer-Verlag, New York, p. 1

Martin S.F., Bilimoria R., Tracadas P.W. 1994, in Solar Surface Magnetism, R. Rutten and C. Schrijvers (eds.), Kluwer Acad. Pub., Dordrecht, Holland, p. 303

Martin S.F. and Mc Allister A.H. 1996, in Magnetodynamic Phenomena in the Solar Atmosphere, (eds.) Y. Uchida, T. Kosugi and H. Hudson, Kluwer Acad. Publ., Dordrecht, Holland, p. 497

Martres M.J., Michard, R., Soru-Escaut, I., 1966, A\&A, 29, 249

Molodij G., Rayrole J., Madec P.Y., Colson F. 1996, A\&A Suppl. Ser., 118, 169

Pevtsov A.A., Canfield R.C., Metcalf T.R. 1995, ApJ, 440, L109

Poland A.I. 1990, Dynamics of Quiescent Prominences, V. Ruždjak, E. TandbergHanssen (eds.), Springer-Verlag, New York, p. 120

Priest E.R., Hood A.W., Anzer U. 1989, ApJ, 344, 1010

Raadu M.A., Schmieder B., Mein N., Gesztelyi L. 1988, A\&A, 197, 289

Ridgway C., Amari T., Priest E.R. 1992, ApJ, 385, 718

Rompolt B. 1990, Hvar Obs. Bull. Vol. 14, 1, 37

Rust D.M., Kumar A. 1994, Solar Phys., 155, 69

Schmieder B. 1990, in Dynamics of Quiescent Prominences, V. Ruždjak, E. TandbergHanssen (eds.), Springer-Verlag, New York, p. 85

Schmieder B., Raadu M.A., Wiik J.E. 1991, A\&A, 252, 353

Seehafer N. 1990, Solar Phys., 125, 219

Shelke R.N., Pande M.C. 1983, Bull. Astr. Soc. India, 11, 327

Srivastava N., Ambastha A., Bhatnagar A. 1991, Solar Phys., 133, 339

Steele C.D.C., Priest E.R. 1992, Solar Phys., 140, 289

Tang F. 1987, Solar Phys., 107, 233

Titov S., Priest E.R., Démoulin P. 1993, A\&A, 276, 564

Uchida Y. 1981, in Proceedings of the Japan-France Seminar on Solar Physics, (eds.)

F. Moriyama, J.C. Hénoux, p. 169

van Ballegooijen A.A., Martens P.C.H. 1989, ApJ, 343, 971

van Ballegooijen A.A., Martens P.C.H. 1990, ApJ, 361, 283

Vršnak B., Ruždjak V., Rompolt B. 1991, Solar Phys., 136, 151

Weiss L.A. et al. 1996, A\&A, 316, 384 